a rilajor purpuse ur ure I ecinilcal Information Center is to provide the broadest dissemination possible of information contained in DOE's Research and Development Reports to business, industry, the academic cornmunity, and federal, state and local governments.

Although a small portion of this report is not reproducible, it is being made available to expedite the availability of information on the research discussed herein. 
$L A-U R--B 8-3320$

DE89 002047

TITLE INTERMEDIATE-ENERGY HADRON INTERACT LONS, II

AUTHOR(S) Richard R. Silbar, T-5

Sugmitted to Summary Talk at Spin and High Energy Physics Sympostun, Minneapolis, MN, September 16, 1988

\section{IDISC 'I.AIMI.K}
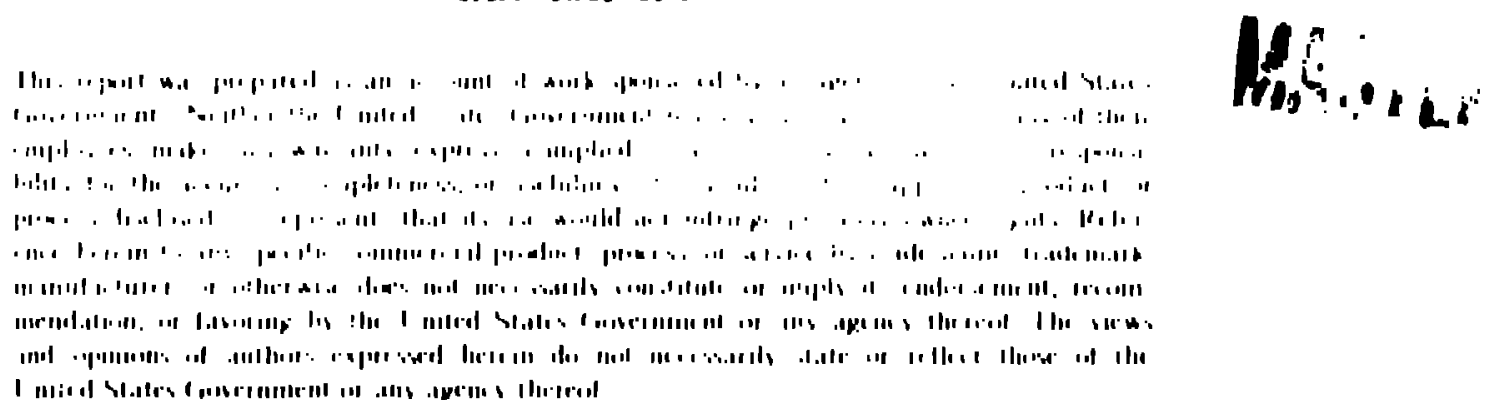

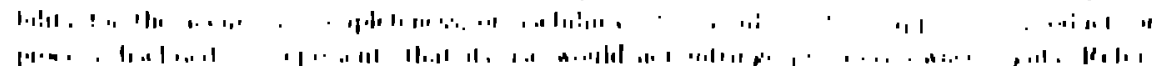

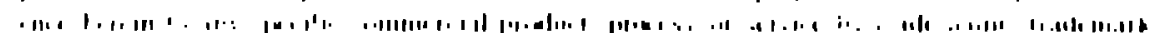

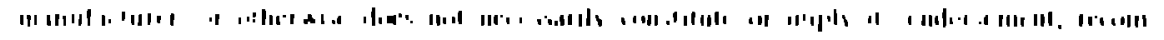
In

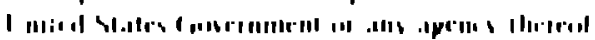

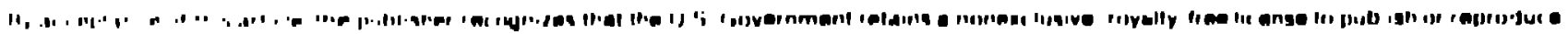

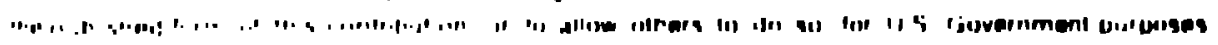

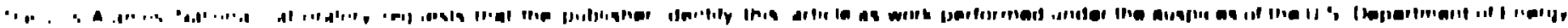
-..- - - - .

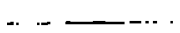




\title{
INTERMEDIATE-ENERGY HADRON INTERACTIONS, II
}

\author{
Richard R. Silbar \\ Los Alamos National Laboratory. Los Alamos. N.M 87545
}

\section{INTRODUCTION}

I will summarize here the second parallel session on intermediate energy hadron reactions, held yesterday afternom. There was already such a summary given Tuesday mornung by Mike Moravesik for a similar session held on Monday afternoon. In the session yesterday there were nine talks in all, six of then hy experimentalists and t!ree hy theoreticians. Both paralled sessions were orfanized (in parallel) by Hal Spinke and Giamni Pauletta, and there was some overlap between them in topies. In the interest of maintaining a perspective. therefore, I will not rest.rict my remarks only to yesterday's talks.

The topies to be covered are as follows. I'll begin with new developments in $. V N \rightarrow . V N \pi$ reactions. This will provicle a natural lead-in to the main topic of this talk. which is dibaryons. This will be followed by disclussion of elastic proton-denteron and inelastic proton-alpha scattering. Then there will be a brief mention of two technical developments. Finally. Ill close by giving short remarks about two peculiarities that were found hy theorists l(x)king at strong interaction amplitudes.

\section{NEW $. V \rightarrow V . V \pi$ I)ATA AND ANALYSIS}

iwo experimentia contributions described new results in intermediate. energy single pion production. There his been an enormons inscease in the last

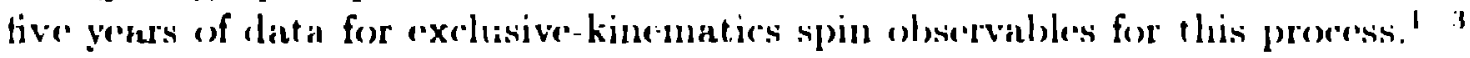
The t wo experiments reported here will in themselves constiture a large fraction of the expanded .V.V $\rightarrow$. V.V $\pi$ data basie.

Peler Riley described work ar. L.AMl'F which meistured spin correlations

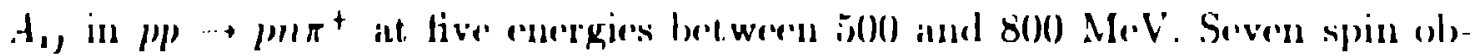
servalbles were measured. Becanse of the polarized proton target. all three tinal state particles were defected to extract a very reban sample of events of alout a million events, distributed exenly over the energy lange. A shent report of this "xperiment his alrebly appented."

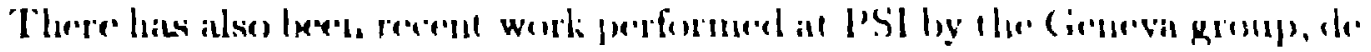

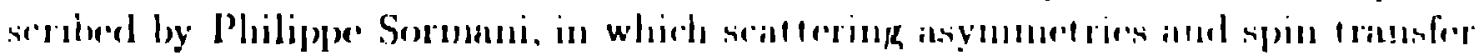

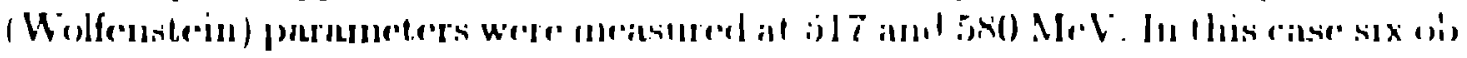

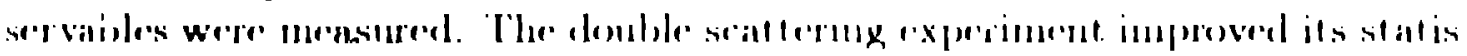

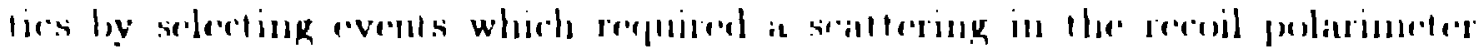

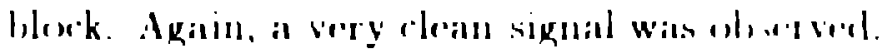

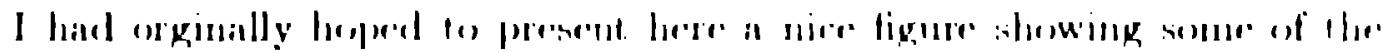

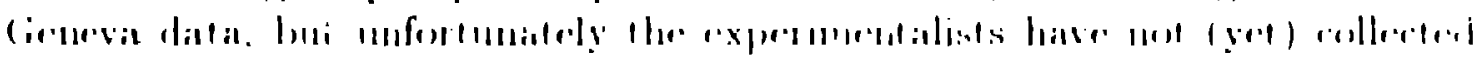


their data in such a form. What is available are (rather dense) tables of their results. This illustrates ine of the difficulties of presenting results for at threebody tinal state: there is a lot of phase spate covered by such data. Sormani gave a waming, and it is one that I ingere with. that presenting one-dinentsional projections of such data can hide a lor (or too much) of the physics present in lhe exclusive data.

With regard to the analysis of the $. Y . V \rightarrow X J$ dat a. I am happy to tell gom that we have now entered a new era. Riley also reported the first partial wase analysis for a $\mathrm{XV} \rightarrow \mathrm{V} \rightarrow \mathrm{V}$ त reaction. The major credit for this achievement goes to Rick Shypit and David Bugg.

What Shypit and Bugg dici an be described briety an follows. They performed a least squares tit for the magnitudes and phase's of the low- $L . X . D$. is partial wave amplitudes. The high $L$ amplitudes were taken from theor: given essentially by the one-pion-exchange Born approximation. and were beld fixed is the fit ting procedure. The model take's account of the isobars $J^{++}, J^{+}$. and $S$ (a mixture of the $S_{11}$ and $S_{31} \pi . V^{2}$ interations). all mutually interfering and all varying appropriately as their invariant masises change. The fit was dome. at each energy, in terms of Gottfried-Jackson variables with the experimental data sorted into about 300 bins.

The extracted phases can. in a weak-conpling limit and after some inanipulation, be interpreted as scattering phase shifts for elastic . V $\rightarrow$ V V scattering. Such information has not heretofore been available. Noreover. the titted nagnitudes, in turn, will som be used to determine the . N.T inelasticity parameters. yls.s. The latter guantities should be mucin more precise than those obtained from elastic $N . N$ phase shift analyses, simply beranse much more detail of the

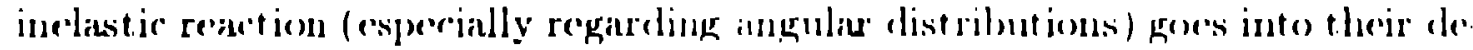
termination.

The tirst partial wave analysis for. $\mathrm{V} J \rightarrow \mathrm{M} J$ is obvionsly not the last one. In the future such andyses should be bissed on an expanded! database which includes all the available data, not just ihat from one experinent. Eventually it will be important to do a urefied analysis of all the re levant reatetions, $N V$.

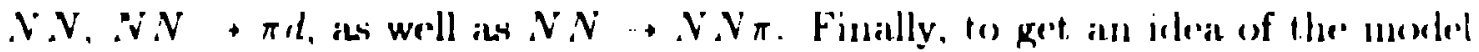
dependence of the results, it will be very useful to see stleh analyses done by more than one investigntor.

\section{DIBAIRYONS AGAIN}

In some perple's minds, the tromble with diharyous is that they mever die. From what has: hapeneel at this conference, it serms that they are not even

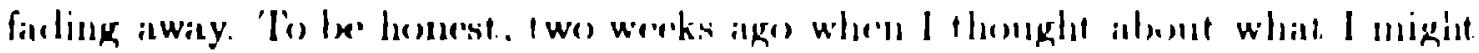

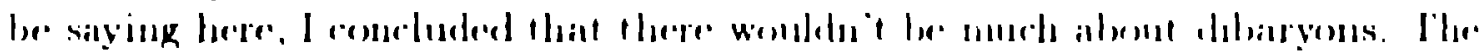

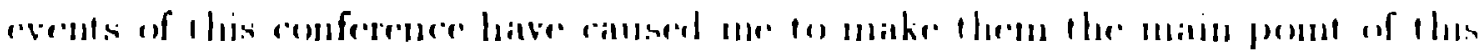
summary.

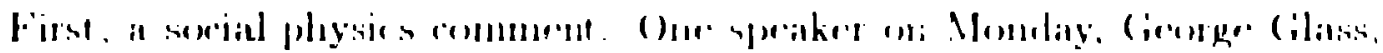

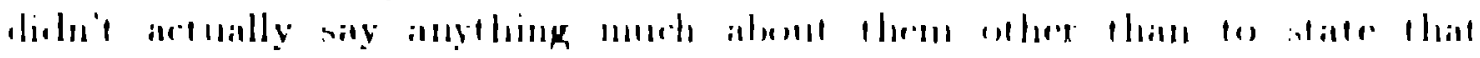


"dibaryon" is a dirty word." He didn't say why. Let me point out that if that is true, the's of is the first eight-letter dirty word anyone ever tanght me. Perhaps that means it is twice as potent as the usual such words.

To make some sense of this 12 year-old subject, it is useful to point ont that there is no real consensus as to what a dibaryon is. This week we have heard the iollowing different working definitions:

- Bugg: "A broad dibaryon corresponds to a phase shift in the . V $>\rightarrow . . \Delta$ channel which rises through $900^{\circ} . "$

- Hoshizaki: "It is a pole in the s-plane."

- Lomon: "An exotic dibaryon is one whose structure dependis explicitly on quark or gluon degrees of freedom."

Please bear these semantic ambiguities in mind as I go throngh what has hap) pened this werek.

To begin. some classification. I'll start the discussion with what is new regarding the broad dibaryon candidates. These are the "old" one's that are seen as loxps in the Argand diagrams for the ${ }^{1} D_{2}$ and ${ }^{3} F_{3} V X \rightarrow . V N$ partial waves (and perhaps others). Then I'll go into the "newer" narrow dibaryon candidates, where by "narrow" I mean something with a width of $30 \mathrm{MeV}$ or less. These seern to be falling into two groups, corresponding to center-of-mass energies $W$ near 2.1 and $2.7 \mathrm{GeV}$.

The broad dibaryon Hap at chis conference arose because the Shypit-Bugg partial wave analys: ; for $N V \rightarrow N \Delta$ mentioned in the last section was pullished with the title "Evidence Against Broad Dibaryons". The extracted clastic $N \Delta \rightarrow . V \Delta$ phase shifts were all rather small (as for the ${ }^{3} P_{2}$ or ${ }^{3} P_{3}$ waves) or falling as a function of energy (as for the ${ }^{5} S_{2}$ wave). Noither rase lox)ks like a rising phase shift assoreiated with a resonance or bound state pole in the $N N \rightarrow . V \Delta$ channe!, which is the dominant channel. In fact. the falling behav ior of the " $S_{2}$ phase shift fits in very nicely with the interpretation of the " $D_{2}$ "dibarycon" as an effert due to the cut from the VJ threshoold."

Riley's talk was inmediately followed by a contribution from N. Hoshizaki. communicaled to this merting by his colleggue, A. Masaike. Hoshizaki's main point is that the result for ther " $S_{2}$ phase shift by Shypit and Bugg could in fact be better interpreted ats evidenee for a broad dibaryon, not against. He considered two moxtels, one involving an inclastic virtual state pole and the other involving an unstable bound state pole. (By way of analoky, recoll the

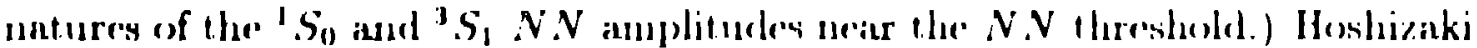

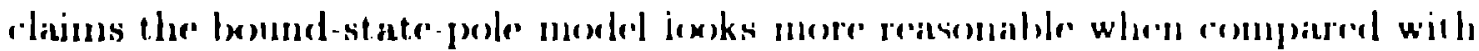
the data.

So, Hat respens what might have beren considered al elesed controversy.

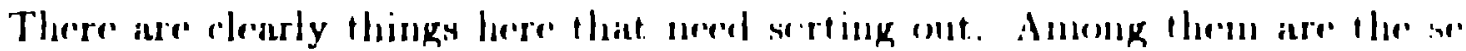

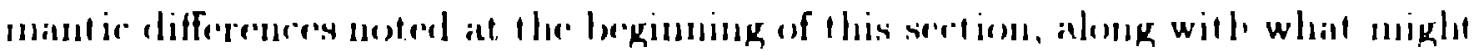
he some mistanderstandings nt the terhuienl level of how the fitting was done.

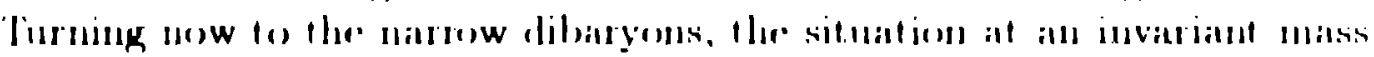

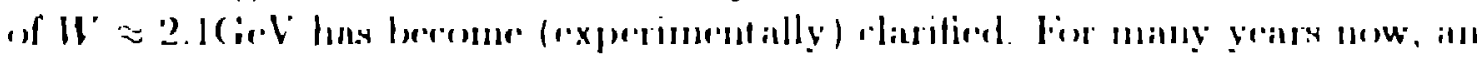


Orsay group ${ }^{\circ}$ has becn seeing three narrow bumps (on top) of a large backgroumd)

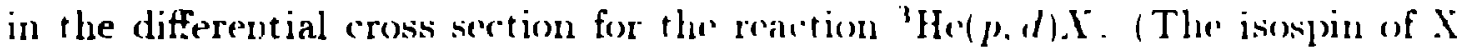
must necessarily be 1.) I recall asking at the Marseille's conference four years ago why' no one from the (Orsay group spoke about the'se interesting datil. The antswer was, "But this is a sin conference and we didn't measture any polariantions."

This week Magid Gazzaly reported that a Minnesenta-line-Trxas-LAMPF group has repeated the Orsay experinuent. but with a polarized proton beam. They confirm the existence of the narrow bumps. In fact. the asymmetry, $A$.. which Moravesik showed you on Tuesrlay. is striking. It shows not only the three ()rsay bumps but two more at lower energies:

$\begin{array}{cl}\text { LAMPF } & \text { Saturne } \\ 2.016 \pm 0.005 & \\ 2.054 \pm 0.004 & \\ 2.125 \pm 0.003 & 2.124 \pm(0.003 \\ 2.152 \pm 0.004 & 2.155 \pm ? \\ 2.181 \pm 0.005 & 2.192 \pm 0.003\end{array}$

All widths in both experiments are $\leq 35 \mathrm{MeV}$.

What can be said at this point alosut these narrow poaks? In the first place you should be aware that as yet there is no spin-parity information about any of these peaks. Nonetheless, as many theorists have conjectured, the above energy levels look like they constitute a rotational band, $W=M_{0}+M_{1} J(J+1)$. Ten vears ago in a calculation based on a stretched six-quark hag model, Mulders, Aert\%. and deSwart ${ }^{\top}$ found just such a formula. In the time since then, however, Mulders has argued that the states they predicted night not be observable."

In a paper presented just after Gazzaly spoke, H. Konno reported a ral. culation done with Nakanura and Noya based on a chuster model with three diquarks. Such a model also gives tive rotational-like states, and it is interesting that these authors were aware of the ()rsaly peaks but not the two bower peaks that had just been reported hy Gazzaly! (One should also note that K. Althoff has just. reninded us, earlier this morning, that one of these lower peaks wiss probably seen at Bom some years ago in the invariant pp mass in the reaction $i d+p p \pi$

A note of caution: the subject nuder cliscussion is a complieated fond borly renction. It combl be that these peaks are not dibaryonts at all but reftert some romplicaterl fow boly final state iateraction. There is no theoretion work that I know of. loowever, that makes such a elain. If the Bonn peak mentioned hy

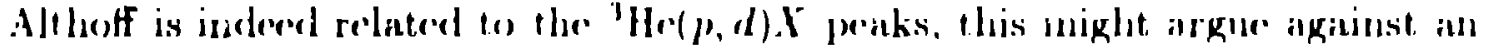
F.S.I. interpretation.

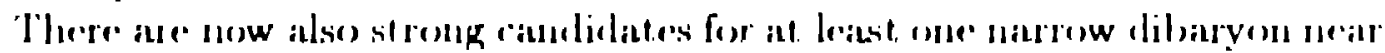

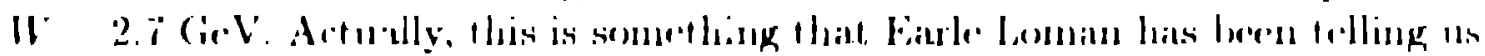
for years, and the subject of his talk here wits where and how to find surth exolic

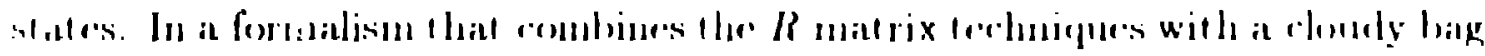

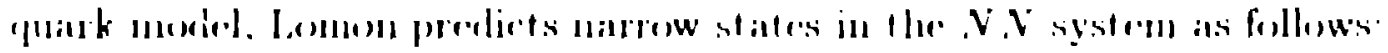




$\begin{array}{lcc}H\left(\mathrm{GeV}^{\circ}\right) & \Gamma(\mathrm{M} \times \mathrm{V}) & J^{\pi} \\ 2.63 & 50 & 1^{+} \\ 2.70 & 50 & 0^{+} \\ 2.58 & 100 & 2^{+} \\ 2.90 & 100 & 3^{+}\end{array}$

Some of these are $I=0$ objects and some are $I=1$. Lomon warns us that the structures associated with these states that can be seen in spin obserwables might only show up as small excursions from the smooth background energy deperdence. Incidentally, Lomon also discussed predictions for strange dibaryon exotics and $2^{*}$ 's. However. nothing was said about the experimental status of such exotics in the intermediate-energy parallel sessions of this conference.

There have been some hints that such V.N exotics may ex:st in this energy region. There is a bunp in the $\Delta \sigma_{l}$, and $A_{l} l$, observables measured long ago at the ZGS, ${ }^{9}$ and more recently, at Saturne, people have seen structure uear 2.7 $\mathrm{GeV}$ in the asymmetry for $p p \rightarrow d \pi^{+} .{ }^{10}$ Nore convincing evidence has emerged during this conference, as reported by A. de Lespuen for the Suclay N.V group headed by François Lehar. For some time now this group has heen measuring three-spin correlations in $\overrightarrow{p p} \rightarrow \overrightarrow{p p}$ srattering. With about 15 such spin observable's at 11 energies for $T_{\text {lab }}$ from 0.853 to $2.7 \mathrm{GrV}$, they have recently ${ }^{1}$ been able to make a direct reconstruction of the five (Wolfenstein) spin amplitudes. What is very interesting about these amplitudes is that three of the four relative phases show a very large dip or jump at one, and only one, energy, $T_{\text {lab }}=2.1$ Gel (which corresponds to $W=2.74 \mathrm{GeV}$ ). Unfortunately, the energy sparing in this region is somewhat coarse; thus one cannot say wehether this is actually 9 insrow structure.

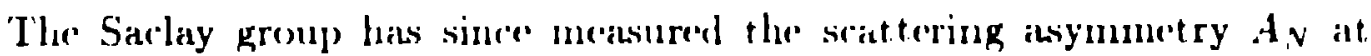
finer energy intervals:

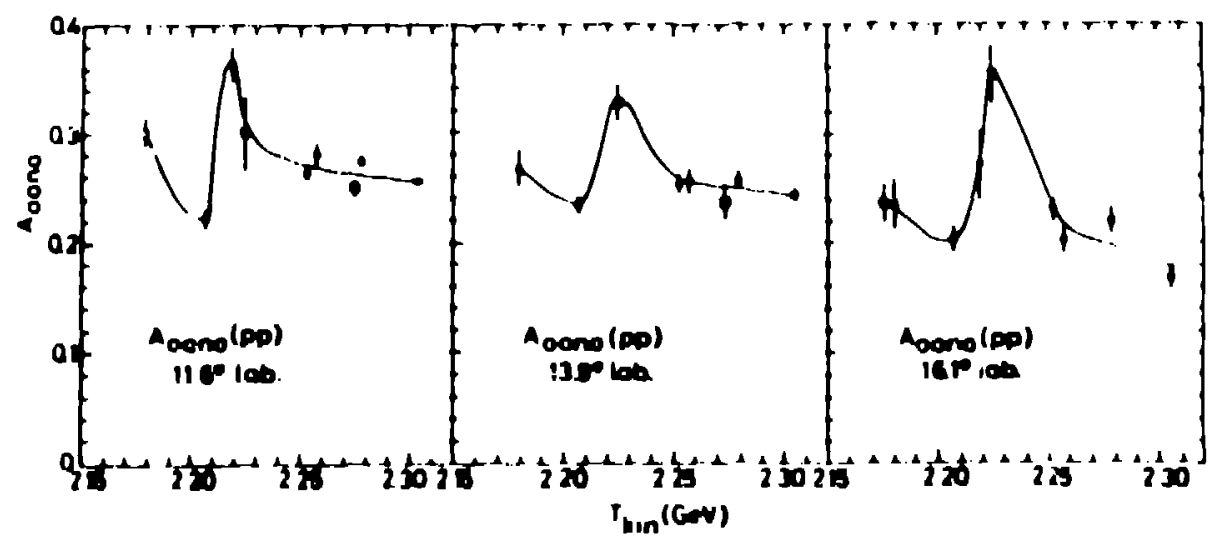

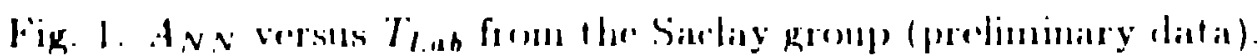

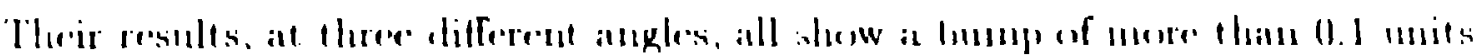

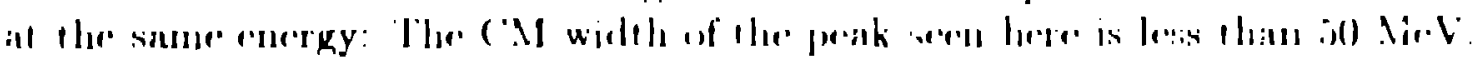


Again, there is no infor ation as to the spin and parity of thes resonamed if it is truly a resonance.

To summarize the present status of dibaryons:

1) The broad dibaryon candidates are still in an unsettled state. The partial wave analysis that led to "evidener a gainst." clearly needs refinement and an expanded database.

2) The narrow bumps seen in ${ }^{3} \mathrm{He}(p, d) \mathrm{X}$ have been continned. There are now five such structures.

3) There is new and pretty convincing evidence for at least one narrow dibaryon near IV $=2.7 \mathrm{G} \cdot \mathrm{l}$.

There will certainly be more discussion of dibaryons at Spin 'O()!

\section{$p d$ AND $p$ SCATTERING}

For some time now there has been an extensive program at the ZGS. LAMPF. KEK, and Saturne to measure spin observables for elastic pd scattering. The leader of this effort has been (ienge Igo, who reported here on the Saturne measurements, done in collaboration with the Lehar group. Here they make good use of one of Saturne's techuical advantages by working with a vector- and tensor-polarized deuteron beam.

The data Igo presented was taken at a deuteron beam energy of 1600 $\mathrm{MeV}$, corresponding to an equitalent proton beam energy of $800 \mathrm{MeV}$, thereby romplementing the LAMPF data set. By now there exists at this energy an "overcomplete" set consisting of about 30 difierent spin observables. An example of their acsults is:

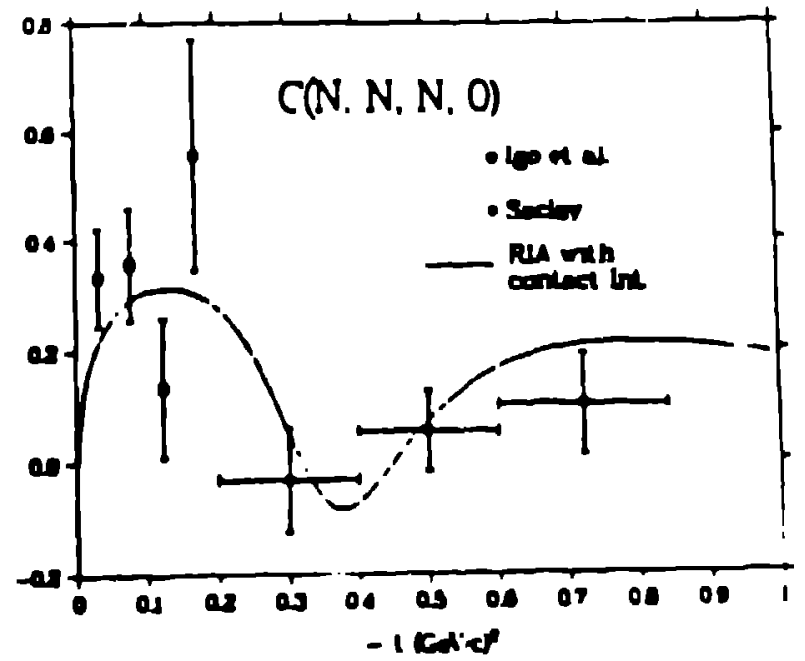

Fig. ". The correlation for pli,p)d, all spins nommad.

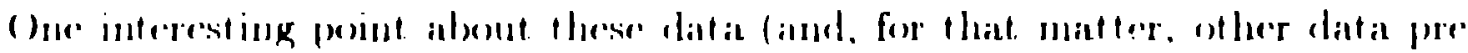
sented at this confenence) is that some of these observables are three spin men

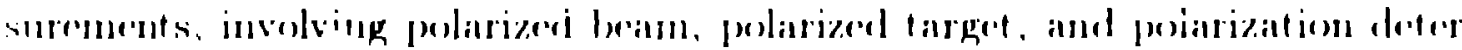
munation of one of the inal particles, including the one shewn in Fig. 2. 'This 
is the first Spin Conference. I believe. in which such three-spin data have been shown and discussed as common st ate-of-the-art results.

What is the motivation for this enormous experimental program? One of Igo's stated reasons is to use the overcomplete set of data 10 construct directly the 12 spin amplitudes for the $p d \rightarrow p d$ reaction. (This bas not yet been done. even at $800 \mathrm{MeV}$ where the data is most complete.) However, I do not find that a very compelling argument: the five clastic $\mathcal{N} . V$ spin amplitudes are quite useful as inputs to nuclear plyysics. but there does not seem to be a comparable usefulness for $p d$ spin amplitudes. Other people. such as Steve Wallace, feel these $p d$ data provide a very useful testing ground for nucleon-nucleus scattering theories. In this case the spin-1 nature of the target is sensitive to details not coming into scattering on the more usual spin-0 nuclear targets. A potentially very important notivation for this $p d \rightarrow p d$ program is. I believe, the possibility of being able to use these data to get good, solid information about the off-shell $V . V \rightarrow N N$ amplitudes. Recall that, despite great effort over twenty years, the proton-proton bremsstratilung measurements have not given us much useful information.

But, can present-day (or near-future) theory confront these data? I am worried that it might not and that these beautiful data may have been taken in vain.

To estimate the amount of effort needed to extract useful $V V$ information from the knowledge of $p d \rightarrow p d$ scattering, let me describe brictly a very recent calculation done by the Graz group. ${ }^{12}$ They work at $T_{\text {l,ab }}=2.5 \mathrm{MeV}$, an energy below the break-up threshold, starting with a separable approximation (up to rank 6) for the Paris $N N$ potential. At this low an energy they can get by with keeping only $N N$ interactions in the ${ }^{1} S_{0},{ }^{3} S_{1} /{ }^{3} S_{1}$, and all $P$ partial waves. (This still means they have up to 16 coupled channols to deal with in any partial wave.) This low-energy problem is plagued by Coulomb complications. but they have learned how to get past this by using a shielded potential in a renormalization approach. The rather creditable results they get for about six observable:; required $600 \mathrm{CPU}$-hours of VAX-8700 time (their estimate). The theoretical effort. I would guess, must have , ost about five man-years.

How would this srale up to a comparably serious effort at $800 \mathrm{MeV}$ ? First. there will be many more partial waves involved, which brings in a factor of 10 or so in CPI-time. The complications of break-1up alone will probably cost a fartor of 2 in CPU-time and double the coding effort. At this point it becomest - lear this is not a problem for a VAX hut for a supercomputer. 'The $10 \times 2 \times$ 600) VAX hours might redure to something like 6(0) hours on a scalar mathiue. Vectorization might save another factor of 10 in ('Pli-time, but it womlel also probably domble the cosling effort.

One big simplitication at son) Mel' is that. one can neglece the ('onlomb

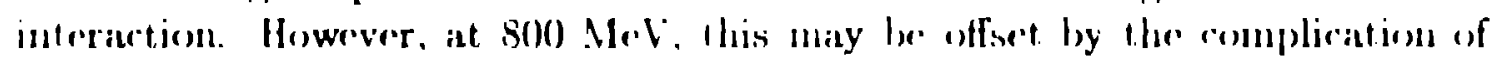
pion production. In the much simpler $. V . V+V . V \pi$ rase, Francesca Sammar rura just spent about three years geetting to the point where she could believe 
the results coming out of her three-body model code. ${ }^{13}$ Maybe, if we are lucky (because the two nucleons in the deuteron are so far apart and we require a pd final state), intermediate $\Delta$ s will decay into (real) pions before they can convert back to nucleons in the next scattering. This possibility needs justification. Another possible simplification is that maybe one can work with a less pathological potential than the one the Graz people used. This too is probably balanced by a lack of knowledge (or consensus) about what the $N . N$ potential model at 800 $\mathrm{MeV}$ is. Or even what it ought to be; there are problems having to do with relativity that we haven't solved yet.

What the last two paragraphs show you is how hard it is to make a good estimate of how long it will take to do a big calculation. It appears that the computing demands are costly but noi unreasonable - of the order of 100 supercomputer hours. The manpower cost, on the other hand, is very large, perhaps 20 to 30 man-years. It is not just someone's $\mathrm{Ph}$. D. thesis.

In the n eantime, there may be simpler things that theoreticians can do to deal with this $p d \rightarrow p d$ data. Indeed, the curve in Fig. 2 is a calculation, ${ }^{14}$ albeit somewhat ad hoc, that doesn't do too badly. Perhaps it is even barking up the wrong tree for me to insist that the problem be done in a partiai wave formalism with the full machinery of a threc-body formalism.

Turning to a subject more in nuclear physics, Sirish Nanda showed us the foilowing picture:

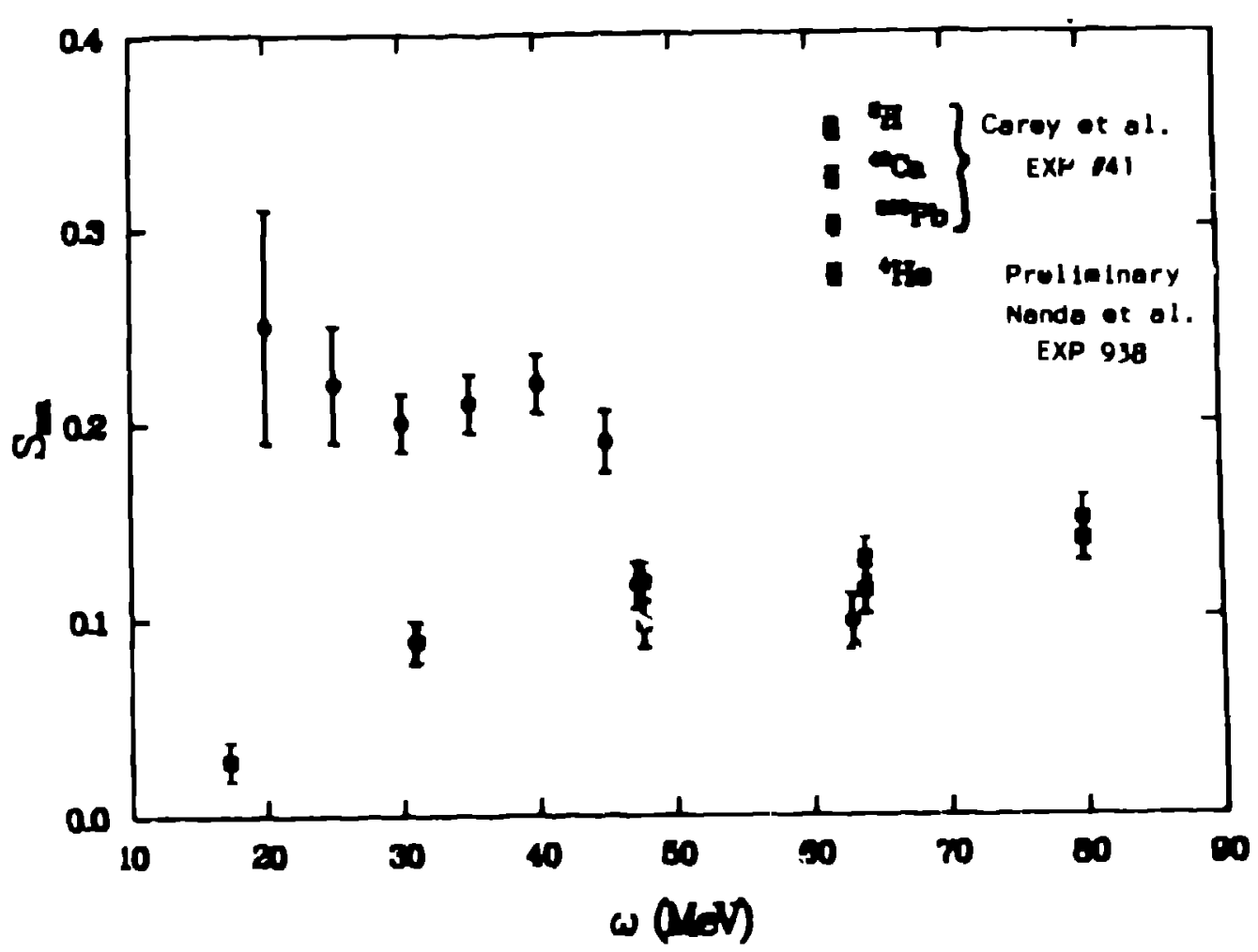

Fig. 3. Spin flip probablities for inelastic proton sorattering 
This is an unexpected result for another fe'w-muleon reartion. ${ }^{4} \mathrm{He}\left(\vec{p} . p^{\prime}\right)^{4} \mathrm{He}{ }^{*}$ at $500 \mathrm{MeV}$ and at a momentum transfer of about $350 \mathrm{Mel} / \mathrm{c}$. The MinnesotatCEBAF-Rutgers-LAMPF group measured the spin-flip probability, $S_{\mathrm{v} . v}=$ $\frac{1}{2}\left(1-D_{v . v}\right)$, with an eye to learning the $\Delta S=1$ response of the ${ }^{4} \mathrm{He}^{2} \mathrm{n}^{\prime}$ : cleus. (Here $\Delta S=1$ refers to spin-Hip, not to a strangeness-changing decay.) The $S_{. v .}$ observable had previously been measured by another group for the nuclear targets ${ }^{2} \mathrm{H},{ }^{40} \mathrm{Ca}$. and ${ }^{208} \mathrm{~Pb}$. What is found (Fig. 3 ) is that once again ${ }^{4} \mathrm{He}$ is different; this time its $S_{\mathrm{N} . \mathrm{V}}$ is about twice the size of that for a "normal" nucleus. As far as I know, there is no explanation for this difference.

\section{TWO TECHNICAL DEVELOPMENTS}

Gerald Gaillard of Geneva described progress in the development of a polarized neutron beam at PSI. They get longitudinally polarized neutrons from longitudinally polarized protons using the ${ }^{12} \mathrm{C}(\vec{p}, \vec{n}), \mathrm{X}$ teaction at $580 \mathrm{MeV}$. The neutron polarizations range from 30 to $45 \%$ and their energies range from 300 to $580 \mathrm{MeV}$. The project has recently been funded to move to a more permanent home on the PSI experimental flox. At the time the project come's to completion (1990), the polarized neutron beam there will have an intensity about fifty times that at LAMPF.

The Geneva group will measure spin observables for elastic np scattering. They have already taken data for asymmetries $A_{N}$ and spin-spin correlations $f_{i j}$. After recommissioning, they plan to measure thee-spin observables.

At the synchrophasotron at Dubna, as those of you who were at the Serpukhov conference alreary know, it is now possible to arceleraie polarized deuteron beans up to about $10 \mathrm{GeV} / \mathrm{c}$. P. V. Nomokonov described two experiments done with this beam. (I characterize these experiments as being under the heading of technical development. although the experiments themselves did have some physics motivation.) In both experiments the bean of $0.5 \times 10^{9}$ dentcrons per cycle with a polarization on the order of $30 \%$ impinges on a $\mathrm{CH}_{2}$ internal rarget. The quantities which were measured were both for the reaction ${ }^{12} \mathrm{C}(d, p) X: A_{N}$ at $T_{L a b}=1.6 \mathrm{GeV}$ and $T_{20}$ at $p_{\text {inc }}=9 \mathrm{GeV} / \mathrm{c}$. The motivation for the $1.6 \mathrm{GeV}$ data was the series of very backward (inclusive) scattering experiments done 10 years ago at LAMPF. ${ }^{15}$

\section{TWO PHENOMENOLOGICAL ODDITIES}

() the theoretical side of the se'ssion, there were, in addition to Loman's talk on exotics, two presentations. The first of these was by Alex Gersten and, in a sense, was a continuation of sone things he already told us at Marseilles. Gersten has found that intermediate-energy $V . V \rightarrow . V . V$ double-spin Hip helicity amplitudes show an astonishing scoling property. Farlier it was a particular combination of isospins for the ampliturle $\langle t+|T|--)$ that scaled (from to) (o) $8(0)$ MeV), loxoking very much like a one-pion exchange Born approximation prediction. Now he has found that a similar scaling applies to the $n p$, pn 
amplitude $(+-|T|-+)$. He is then able to fit these amplitudes in a very simple "no exchange model", that is. by using OPE scattering wave functions outside some radius $R$ (which is $\approx 1 \mathrm{fm}$ ) and setting the wave functions to zero inside $R$. This seems to imply that these double-spin-Hip amplitudes to not involve any short-range forces!

It would be very interesting to see if this scaling behavior also holds at higher energies. The combinations Gersten forms all require knowing the $n p$ as well as the $p p$ amplitudes, and the former are a bit of a problem right now. As mentioned earlier, there are 11 higher energies for which the Saclay NN group have determined the $I=1 \mathrm{pp}$ spin amplitudes. Earlier measurements at the ZGS, at $6 \mathrm{GeV} / c$, also determine a complete set of $p p$ amplitudes.

In a somewhat different vein, but in much the same spirit. Mike Moravesik described a phenomenon which he and Gary Goldstein have found when strong interaction amplitudes are recast into the so-called "planar-transverse" representation. What they find is that these amplitudes have a strong tendency to be all purely real or purely imaginary, relative to one another. This has been found to be true for elastic $N N \rightarrow . N . V$ scattering (where the effect is more pronounced above $300 \mathrm{MeV}$ ), for $\pi N \rightarrow \pi . V$ (for energies up to $40 \mathrm{GeV}$ ), and (with some theoretical input) for $\pi d \rightarrow \pi d$.

Both these talks point out some very peculiar properties of strong interaction amplitudes. I don't think anyone, at this point, knows the reasons for, or the implications of, these phenomena. Perhaps one or another of these oddities will provide an important clue that leads to new physics.

\section{GEFERENCES}

1. A. D. Hancock et. al., $t_{N}$ in $p p \rightarrow p n \pi^{+}$, Phys. Rev. C 27, 2742 (1983).

2. C. L. Hollas et al., $D_{i j}$ in $p p \rightarrow p n \pi^{+}$, Phys. Rev. Lett. 55, 29 (1985).

3. P. J. Riley et al., $D_{i j}$ in $p p \rightarrow p p \pi^{0}$, Phys. Lett. 197B, 23 (1987).

4. R. L. Shypit et al., Fhys. Kev. Lett. 60, 001 (1988).

5. E.g., W. M. Kloet, J. A. Tjon and R. R. Silbar, Phys. Lett. 99B, 80 (1981).

6. E.g., B. Tatischeff, Phys. Lett. 154B, 107 (1085).

7. P. J. G. Mulders et al., Phys. Rev. Lett. 40, 1543 (i985).

8. P. J. G. Mulders, Phys. Rev. D 25, 1269 (1085).

9. 1. P. Auer et al., Phys. Rev. Lett. 61, 1077 (1988); Phys. Rev. D 34, 2581 (1986).

10. R. Bertini et al., Phys. Lett. 162B, 77 (1985); Phys. Lett. 203B, 18 (1988)

11. C. D. Lac et al., submitted to J. de Phys. (Paris), June 1988.

12. G. H. Berthold et cl., Phys. Rev. Lett. 61, 1077 (1988).

13. F. Sammarruca, Ph. D. thesis, Virginia Polytechnical Institute and State University, August 1988.

14. E. Blesczynski et al., AIP Conf. Pror. 150, 1208 (1986).

15. S. Frankel et al., Phys. Rev. Lett. 41, 148 (1978). 